\title{
Modeling the Rising Tails of Galaxy Rotation Curves
}

\author{
Fan Zhang ${ }^{1,2}$ (D) \\ 1 Gravitational Wave and Cosmology Laboratory, Department of Astronomy, Beijing Normal University, \\ Beijing 100875, China; fnzhang@bnu.edu.cn or fnzhang@mail.wvu.edu \\ 2 Department of Physics and Astronomy, West Virginia University, PO Box 6315, \\ Morgantown, WV 26506, USA
}

Received: 9 November 2018; Accepted: 22 January 2019; Published: 28 January 2019

\begin{abstract}
It is well known, but under-appreciated in astrophysical applications, that it is possible for gravity to take on a life of its own in the form of Weyl-curvature-only metrics (note that we are referring to the Weyl-only solutions of ordinary General Relativity; we are not considering Weyl conformal gravity or any other modified gravity theories), as numerous examples demonstrate the existence of gravitational fields not being sourced by any matter. In the weak field limit, such autonomous gravitational contents of our universe manifest as solutions to the homogeneous Poisson's equation. In this note, we tentatively explore the possibility that they may perhaps account for some phenomenologies commonly attributed to dark matter. Specifically, we show that a very simple solution of this kind exists that can be utilized to describe the rising tails seen in many galaxy rotation curves, which had been difficult to reconcile within the cold dark matter or modified Newtonian dynamics frameworks. This solution may also help explain the universal $\sim 1$ Gyr rotation periods of galaxies in the local universe.
\end{abstract}

Keywords: dark matter; galaxies; gravitation; rotation curves; galaxy rotation period

\section{Introduction}

A confirmation of the nature of dark matter (DM) remains elusive. Weakly-interacting massive particles have recently been running up against strong constraints set by the null results of direct [1-3] and indirect [4] detection experiments. Astrophysical observations have also ruled out the original version of axions proposed to solve the strong CP problem [5,6], and searches for its "invisible" reincarnations have also become severely constrained [7,8]. Regardless of particle specifics, the cold DM (CDM) implied hierarchical structure formation paradigm faces a number of challenges such as the angular momentum problem [9], the missing clusters [10] and satellites [11] problems, as well as the issue of missing stochastic gravitational wave background [12]. On the other hand, the modified gravity theories have to overcome well-posedness issues $[13,14]$ and problems such as unstable stars [15] (see also Famaey and McGaugh [16] for other complications with which specific theories have to contend).

Since new physics are not readily forthcoming, it is perhaps worthwhile to revisit the question of whether the proven General Relativity (and its weak field limit), together with the known Standard Model particles, can possibly already account for some of the apparent DM (aDM) phenomenologies. This prospect may be viable due to the presence of autonomous gravitational fields not sourced by any matter, whose existence has in fact been known since the early days $[17,18]$ (see also, e.g., Brill and Hartle [19] or Misner and Taub [20] for a curvature-singularity-free warped universe that is completely devoid of matter; for complex manifolds, we also have the well-studied Calabi-Yau manifolds [21,22]). However, when trying to explain the observation of the mysterious gravitational content of the universe, which does not appear to interact through other forces, this most natural of possibilities 
somehow escaped close scrutiny (perhaps because the now dashed "WIMP miracle" and a desire for discovering new particles drew the discussion to new matter species from the beginning). Through our admittedly preliminary investigations in this paper, we hope to attract more attention to this less exotic explanation of the aDM phenomenology.

Turning to the specifics, we note that autonomous gravity must be carried within the Weyl curvature tensor ${ }^{1}$ (since the Ricci curvature tensor equates to the matter stress energy tensor), which contains not only gravitational waves (loosely, Newman-Penrose pseudo-scalars $\Psi_{0}$ and $\Psi_{4}$ ), but also a Coulomb piece ( $\left.\Psi_{2}\right)$, usually in a difficult-to-disentangle jumble [23]. In other words, autonomous gravity configurations are not always simple nonlinear wave packets that can easily disintegrate. Geometrically, the Weyl tensor represents the variations in sectional curvatures ${ }^{2}$ [24] (and Ricci, their average), so its very presence is indicative of anisotropy and clumping, ideal for seeding structure formation, but it is simultaneously less prone (than CDM, which behaves more like pressure-less dust) to develop shocks or other sharp features [25] (otherwise, vacuum black hole simulations using spectral methods would have unlikely succeeded), as it is governed by the Bianchi identities.

When studying galaxies and clusters, Newtonian gravity is usually adopted. In this language, the autonomous gravity hides in the solutions to the homogeneous Poisson's equation. Specifically, although the metric perturbation component $h_{t t}$ can be equated to an effective Newtonian-style potential $\Phi=-h_{t t} / 2$ in the weak stationary field and slow motion limit, Einstein's equations only reduce to Poisson's equation ${ }^{3} \nabla^{2} \Phi=4 \pi \rho_{\mathrm{M}}$ and not all the way to Newton's law of universal gravitation. A solution to a linear inhomogeneous equation like Poisson's can be constructed out of two parts: a "particular solution" of the inhomogeneous equation, for which $\Phi_{\mathrm{N}}=-\int\left(\rho_{\mathrm{M}} / \Delta r\right) d V$, produced by Newton's law qualifies as one and a solution $\Phi_{\mathrm{H}}$ to the homogeneous version of Poisson's equation $\nabla^{2} \Phi_{\mathrm{H}}=0$ whose utility is to enforce boundary conditions (which need not be trivial in a cosmological context). Such a $\Phi_{\mathrm{H}}$ field, although orphaned (not sourced by any matter), nevertheless generates a gravitational acceleration $-\nabla \Phi_{\mathrm{H}}$ and, when forcibly interpreted through Newton's law, would masquerade as a form of fake matter (henceforth referred to as the effective Weyl matter $(E W M)$; since $\Phi_{\mathrm{H}}$ is not sourced by local matter, it can only contribute to the Weyl half of the reconstructed-by using $\Phi$ as $-2 h_{t t}$-Riemann tensor), which must be dark as it cannot participate in Standard Model interactions.

For the weak field limit, once again recall that the higher order coupling between different sub-components of the overall potential $\Phi$ are negligible, so the governing equation for $\Phi$ is the linear Poisson's equation, for which solutions can be superimposed linearly, thus the EWM clouds are decoupled from matter-generated gravity and each other at leading order (but the matter can see the EWM at this order through the geodesic equations), allowing a dissociation of the gravitational and X-ray luminosity centers in the Bullet Cluster [26]. However, General Relativity (GR) is ultimately nonlinear, so given sufficient interaction time, different EWM clouds and matter-generated gravity can eventually couple at higher orders, possibly contributing to the more complicated post-slow-speed-collision aDM distributions of the Train Wreck [27] (relative velocity at $1077 \mathrm{~km} \mathrm{~s}^{-1}$ vs. Bullet's $4700 \mathrm{~km} \mathrm{~s}^{-1}$ [28]) and, to a lesser extent, Musket Ball [29] (1700 $\mathrm{km} \mathrm{s}^{-1}$ ) clusters.

Further complications arise when one realizes that our universe may not have a full set of non-singular boundaries (e.g., its spatial slices may be large three-dimensional spheres), in which case the boundary conditions for the metric or $\Phi$ become missing or effectively cyclic (which is also not very constraining). However, in full GR, the gravitational fields are endowed with geometric

1 Ordinary matter can also generate this type of curvature, so not all of it is autonomous, but all autonomous gravity is Weyl, because the Ricci tensor equates directly to the matter (incl.cosmological constant) stress-energy tensor.

2 The Gaussian curvature of the various 2D geodetic surfaces developed out of 2D planes in the tangent space of the spacetime at any location.

3 We work under a geometrized unit system where $G=1=c$, with kiloparsecs being the fundamental length unit. 
significances, so additional topological constraints arise to fill the gap (spacetime cannot just bend arbitrarily if it is to close up into the correct topology). Imagine an idealized compact boundary-less universe (similar considerations can be applied to individual spatial slices), whose Chern-Pontryagin density [30] ( $R_{b c d}^{a}$ is the Riemann tensor, $\epsilon$ the Levi-Civita pseudotensor, and $C^{a}{ }_{b c d}$ the Weyl curvature tensor):

$$
\rho_{\mathrm{CP}} \equiv \frac{1}{2} \epsilon^{c d e f} R_{b e f}^{a} R^{b}{ }_{a c d}=\frac{1}{2} \epsilon^{c d e f} C^{a}{ }_{b e f} C^{b}{ }_{a c d}
$$

and Gauss-Bonnet invariant ( $R$ is the Ricci scalar):

$$
\rho_{\mathrm{GB}}=\sqrt{-g}\left(\frac{2}{3} R^{2}+2 R_{a b} R^{a b}-C_{a b c d} C^{a b c d}\right)
$$

integrate into the instanton number [31] and $32 \pi^{2}$ times the Euler characteristic [32], respectively, both of which are small integers for simple topologies. In other words, the average amplitudes of $\rho_{\mathrm{CP}}$ and $\rho_{\mathrm{GB}}$ should be on par with the inverse of the spacetime's volume. However, a cosmological constant $\Lambda$ can create a surfeit of $\rho_{\mathrm{GB}}$ (via $R_{a b}=\Lambda g_{a b}$ ) if the size of the universe is greater than $1 / \sqrt{\Lambda}$ ( $\sim 10$ Gly for the real thing). In order to achieve a recalibration of $\rho_{\mathrm{GB}}$ without inadvertently bloating $\rho_{\mathrm{CP}}$, two new gravitational components should be introduced, at commensurate abundances to each other and to $\Lambda$, so near-cancellations can occur. The EWM and ordinary Standard Model matter are the obvious choices.

Incidentally, this desirability for two separate new ingredients is not apparent when examining the specific Friedman-Lemaitre-Robertson-Walker metric, whose oversimplifying assumption of exact isotropy artificially takes the Weyl half of GR and $\rho_{\mathrm{CP}}$ out of action (there is no way to arrange the principal null directions of the Weyl tensor [33] without breaking isotropy, or equivalently, one can invoke the previous sectional curvature argument). In reality, the anisotropy on small scales is boosted by the two derivatives (multiplications by wavenumbers in momentum space) taking us from metric to curvature, so Weyl is far from negligible even with near-isotropy on large scales. In any case, regardless of whether this balancing act required by the compact example describes our actual universe, there is no reasoning that prevents the EWM from being present in it anyway.

\section{Effective Weyl Matter Phenomenology}

\subsection{Overview}

To execute a preliminary assessment of the admissibility of the EWM as an aDM candidate, we examine the galaxy rotation curves that helped launch the field of DM research in the first place. As the assumptions of a weak stationary field and slow motion (as compared to the speed of light $c$ ) are reasonable, we can simply use the effective potential formalism (recall this is more general than Newton's law) and superimpose the potentials or accelerations from different origins.

Before going into any details, it is worthwhile re-emphasizing that we are not proposing a new modified theory of gravity. We are working completely within GR (and its weak-field slow-motion Newtonian limit). The governing equation that we solve is simply the Poisson equation $\nabla \Phi=4 \pi \rho_{M}$ (whose solution is $\Phi=\Phi_{\mathrm{H}}+\Phi_{\mathrm{N}}$, with $\Phi_{\mathrm{H}}$ being the solution to the homogeneous version of the equation and $\Phi_{\mathrm{N}}$ the particular solution to the inhomogeneous equation) satisfied by the Newtonian gravitational potential, which has dominated gravitational physics for centuries. What we are doing differently is that we also consider those solutions to this equation that have previously been thrown away due to oversimplified boundary conditions (i.e., we are not changing any theories, just trying to make sure that the relevant solutions are not inadvertently discarded).

The potential issue with boundary conditions we explore is that traditionally in astrophysics, it had been second nature to assume that the object in focus can be studied in isolation. For example, when trying to solve the gravitational field inside a star, we often assume that it is sitting all by itself in 
an otherwise empty universe, so that the gravitational field comes only from the star itself and drops to zero when we are far away from the stellar surface (such an asymptotically-vanishing boundary condition ends up setting $\Phi_{\mathrm{H}}=0$ ). This is not always valid of course, since the surroundings of the star may not really be negligible (e.g., the star may be in a binary with a black hole) and may exert strong influences on the interior of the star (e.g., tidally deform it). Inside the star then, such external influences manifest through a non-vanishing $\Phi_{\mathrm{H}}$, since they are not sourced by matter inside of that star. Similarly with galaxies, their rotation curves had traditionally been examined by assuming that the galaxies exist in isolation (i.e., the gravitational field is implicitly assumed to vanish at large distances, thus setting $\Phi_{\mathrm{H}}=0$ ), even though we now know that they must be subsumed into larger-scale gravitational structures like clusters and filaments, so we must have $\Phi_{\mathrm{H}} \neq 0$. In fact, the aDM seen in these larger structures could also be of $\Phi_{\mathrm{H}}$ in nature (i.e., not sourced by the matter content within the clusters, etc.), which are themselves determined by the boundary and initial (since at the largest scales, we cannot assume stationarity) conditions of the universe (please refer to the discussions in Section 1). When zooming in on an individual galaxy then, these clusters $\Phi_{\mathrm{H}}$ serve to produce non-trivial boundary conditions on the region around that galaxy, which we carve out as the computational domain to solve the Poisson equation.

Our new ingredient helping to explain the rising tails of the rotation curves is simply this non-vanishing $\Phi_{\mathrm{H}}$, which has always been allowed by the Newtonian limit of GR (although Newton's law is narrower in scope than Poisson's equation and only gives $\Phi_{\mathrm{N}}$ ), but had unfortunately been thrown away by the implicit assumption that galaxies exist in isolation. When elevated back to GR language, this $\Phi_{\mathrm{H}}$ must be a part of the Weyl curvature tensor, because it is not sourced by in situ matter (the other half of the Riemann tensor, namely the Ricci tensor, must equate to the matter stress-energy tensor and thus cannot correspond to $\Phi_{\mathrm{H}}$ ). Therefore, in the bigger picture, the $\Phi_{\mathrm{H}}$ we consider below in the more specialized context of individual galaxies (explaining galactic-scale $\mathrm{aDM}$ phenomenology) is also an integral part of the autonomous gravitational content of the universe (explaining aDM phenomenology on all scales).

With autonomy, $\Phi_{\mathrm{H}}$ brings with it genuine additional freedom, which means that the present framework is more flexible than modified gravity theories such as modified Newtonian dynamics (MOND), which keeps $\Phi_{\mathrm{H}}=0$ and instead adjusts the dependence of $\Phi_{\mathrm{N}}$ on matter, and therefore does not really add any freedom unless this dependence is allowed to vary from situation to situation. As we have discussed in Section 1, this freedom allowed a decoupling between $\Phi_{\mathrm{H}}$ and $\Phi_{\mathrm{N}}$, and thus consistency with the Bullet cluster observations. Another place where such flexibility may prove useful is when aDM exhibits variations across galaxies, e.g., if the claim of a DM-less galaxy by van Dokkum et al. [34] turns out to be valid, then the present framework can easily accommodate it if the local environment within the galaxy cluster is such that the galaxy in question experiences boundary conditions that lead to a vanishing $\Phi_{\mathrm{H}}$ (i.e., the galaxy is more isolated from its siblings than usual). We caution though that an alternative explanation is that since the EWM only becomes important at large galactocentric radii (see below) and observations of distant galaxies may simply be seeing only the brighter central core, so the lack of aDM may just be that the EWM-dominated regions are not seen.

\subsection{A Galactic Effective Weyl Matter Solution}

Our autonomous gravity component mimicking a galactic aDM halo is the very simple axisymmetric solution (this form provides a rising tail without introducing any singularities or discontinuities in the potential; in particular, the lack of a singularity at the origin fixes the powers):

$$
\Phi_{\mathrm{H}}=\frac{\kappa}{2} \rho^{2}-\kappa z^{2}
$$


to the homogeneous Poisson's equation, which in the cylindrical coordinates $\{\rho, \phi, z\}$ reads:

$$
0=\nabla^{2} \Phi_{\mathrm{H}}=\frac{1}{\rho} \frac{\partial}{\partial \rho}\left(\rho \frac{\partial \Phi_{\mathrm{H}}}{\partial \rho}\right)+\frac{1}{\rho^{2}} \frac{\partial^{2} \Phi_{\mathrm{H}}}{\partial \phi^{2}}+\frac{\partial^{2} \Phi_{\mathrm{H}}}{\partial z^{2}} .
$$

The gravitational acceleration associated with this solution is given by:

$$
g_{\rho}=-\kappa \rho, \quad g_{\phi}=0, \quad g_{z}=2 \kappa z,
$$

and it is simple to double-check that the flux of this acceleration across the surface of any pillbox surrounding the galaxy (the galactic plane is assumed to be at $z=0$ ) vanishes, so there is no mass sourcing it. This gravitational field is supported instead by the boundary conditions where the galactic aDM halo subsumes into the aDM distribution for the entire cluster (note that studies revealing a collusion between the halos of different galaxies in the lensing data [35], in their orientations [36], and on missing aDM in the local universe [37] suggest that these individual halos should not exist in isolation, but instead form an interconnected whole).

It is interesting to note that such boundary conditions (can be recovered by taking $\rho$ and $z$ to large values in Equation (3)) would entail larger acceleration values in the galactic corona, providing a possible mechanism to produce the highly energetic $\left(10^{5}-10^{6} \mathrm{~K}\right)$ gases found in those remote regions. Concentrating on the galactic plane where we have more tracers and data, we note that the component $g_{\rho}$ ( $g_{z}$ will be discussed in Section 2.4) along the plane increases linearly with $\rho$, translating into an "extended DM core" in the CDM terminology (i.e., a constant $\rho_{\mathrm{aDM}}$ spherical density profile; there is thus no cuspy halo problem [38] in the galactic center), which has occasionally been noted in the literature (see, e.g., $[39,40]$ ) to be able to provide better fits to data than CDM-based profiles (e.g., [41,42]). To see the direct effects of $g_{\rho}$, we need to go to very large $\rho$ and study the tails of the galaxy rotation curves, which we turn to examine now.

\subsection{Anatomy of Rotation Curves}

Rising tails at large galactocentric radii $\rho$ are seen for a large number of rotation curves (see the many references in Ruiz-Granados et al. [43]), yet such a trait cannot be explained with the theoretically-modeled CDM profiles, nor MOND [44], which predict curves that are flat or declining at very large $\rho[39,45]$. This is a cause for concern given that the tail is particularly informative, because while uncertainties in ordinary matter distribution (e.g., those associated with stellar mass-to-light ratios and the molecular gas densities) complicate interpretation of the inner and intermediate segments of the rotation curves, their impacts usually do not extend to the far outer regions, which consequently reflect the properties of the aDM in a more transparent manner.

Unfortunately, perhaps precisely because rising tails do not support either CDM or MOND, neither camp has paid much attention to it, especially in the written literature, sometimes chalking it up instead as due to low accuracy of measurements carried out at the extreme galactocentric distances (this is the reason why we included the dwarf galaxy in our Figure 1c below, which shows that the linearly-rising EWM profile is required not just for the tails, but also for the aDM contribution to not overshoot observed curves on the inside, where the accuracy argument does not apply; this issue is most salient for dwarf galaxies since the aDM contribution is appreciable even on the inside for them, so there is not a large baryonic contribution that masks the problem). In other cases, continuously-rising curves are interpreted as "have not yet reached the flat part by the last measured point" (see, e.g., the first paragraph of Section 7.1 in de Blok et al. [46], and also their Figure 57, which in fact displays many curves with rising tails). In other words, it appears that the extreme incompatibility of rising tails with CDM or MOND had prompted many authors to disregard them even as a possibility, and instead unconsciously interpreted them out of existence, even in cases where data for sufficiently large galactocentric radii are available and rising tails are unambiguously seen. 
We have to caution nevertheless though that whether a rising tail is universal across all galaxies has not been firmly established observationally. Exploratory examinations of the extreme outer edges of the galaxies are not receiving sufficient observation time allocations, so it is possible that the absence of a rising tail from some curves may simply be due to data points not extending far enough out (by definition, the rising tails are regions where baryonic matter becomes subdominant, so there are few baryonic tracer objects to measure the rotation speeds with; further in from the tails, an $\sim 1 / \sqrt{r}$ drop-off from baryonic contribution combines naturally with an $\sim r$ rising aDM contribution into a rather flat curve in the transition zone where the two are of comparable strengths). We note in addition that our study can be used to infer an estimate on when the rising tails would appear (since $\kappa$ appears common across galaxies; see below for details), and so, it is easy to verify or falsify with large-scale observation campaigns of galaxy outer regions.

More confusingly, with the existing surveys, people had been more interested in declines in the curves associated with mass distribution cut-offs, so even when data already exhibit early signs of rising tails, they are instead interpreted as "asymptotically approaching a flat curve after a temporary dip" (see many examples of this morphology in the agnostic sample of THINGS curves in Wojnar et al. [47]) and used to argue that earlier results showing declining curves do not provide the full picture, and making observations further out instead produces once again "flat" profiles. We do not quite agree with this "dip" interpretation (which assumes that the curves will flatten off rather than keep rising), because to produce this "rising back up" feature on top of the CDM or MOND profiles (which are smoothly declining or flat at large distances), baryonic matter must form a high density shell at extremely large galactocentric distances, and we have not seen any discussions as to why and how such a shell should form (especially since this morphology occurs quite frequently, the shell must be a ubiquitous robust feature of galaxies), or indeed seen any observational evidence for the existence of such shells at all. We believe that if observational efforts are carried further out to even larger distances, undeniable tails rising above any sensible definition of "flat" would become clear.
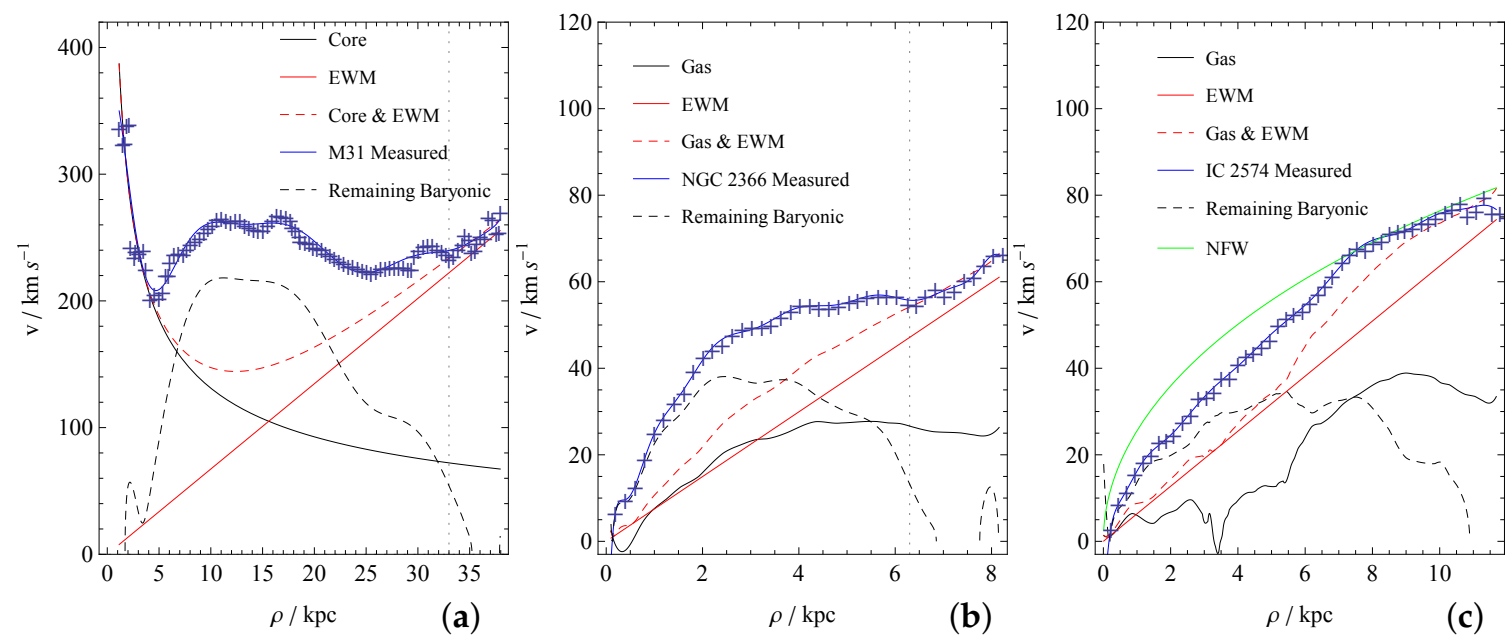

Figure 1. The fitted rotation curves for (a) M31 with $\kappa=5.035 \times 10^{-10} \mathrm{kpc}^{-2}$ and $M=4 \times 10^{10} \mathrm{M}_{\odot}$, (b) the dwarf galaxy NGC 2366 with $\kappa=6.215 \times 10^{-10} \mathrm{kpc}^{-2}$, and (c) the dwarf galaxy IC 2574 with $\kappa=4.5 \times 10^{-10} \mathrm{kpc}^{-2}$. The blue crosses are the Hi -implied rotation speeds taken from Chemin et al. [45] and Oh et al. [48] and the blue curves their polynomial interpolations. The black curves in panels $(\mathbf{b}, \mathbf{c})$ are the measured gas contributions from Oh et al. [48]. The green curve in panel (c) is a CDM profile fit (see Equation (6)) demonstrating that such profiles tend to overshoot the observed rotation curve. Note that the rotation speeds add in quadrature, so accelerations add linearly.

In fact, the EWM model quite naturally produces such apparent "dip" features, as well as the declining curves that constitute another large population of the curves in, e.g., the SPARC sample. Because the EWM's $\left|g_{\rho}\right|$ profile rises linearly in a fashion more slowly than the CDM and MOND profiles, the inside regions of the rotation curves are more dominated by baryonic matter in this model. 
Therefore, when the baryonic matter is fairly concentrated (e.g., into the bulge), the rotation curve would naturally contain a declining mid-section where the baryonic contribution quickly declines (nearly Keplerian), but where the EWM contribution is still relatively small and so has not taken over yet. Only after going further out would the EWM contribution begin to show up. Therefore, depending on the galactocentric radii for which we have good data (as exemplified by truncating the dashed red line in Figure 1a at different places), one may see a (truncated) curve ending up in either a decline (in some extreme cases, the curves may appear to contain no dark matter contribution, which is difficult to explain with CDM or MOND, but quite natural in the present model; the EWM simply has not risen to an appreciable level yet at the observed distance), a "rising back up" convex shape, or a continuously-rising tail that rises above any sense of "flatness". In other words, we think that the EWM model is not only suitable for explaining the rising tails, it is also compatible with the other common morphological features of the rotation curves seen further in.

Coming back to the tail segment that is most reflective of the aDM properties, we note that a rising tail is quite natural with an EWM-generated linearly-rising $\left|g_{\rho}\right|$. For concreteness, we carry out fits for a few example galaxies where high quality data for the rotation curve are available. Before starting though, we caution that there is significant difficulty with fitting to rotation curves, since besides the aDM contributions, there are also other unknowns like the mass-to-light ratio or molecular gas and compact object abundances, as well as more esoteric features such as whether a galaxy has recently gone through a merger so there are baryonic density waves. Even if measurements of "all" relevant quantities are available (which as far as we know is not the case for any galaxy, since at the very least, the molecular gases are difficult to see), they would contain large uncertainties, so it is not quite optimal to produce least squares fitting wellness parameters for entire curves, since the goodness of fits will have to be interpreted conditional on the aforementioned uncertainties associated with all the baryonic parameters. Therefore, the statistical significances of the fits are substantially degraded, rendering the fits much less informative. In short, due to the large uncertainties, there are too many handles in the baryonic models that one can crank to yield essentially any conclusion one wants about the aDM profile.

However, it is possible to make more robust quantitative measurements based on particular segments of the rotation curves. In particular, because baryonic matter density drops with increasing galactocentric radius $\rho$, their contribution to the overall rotation curve must rapidly decline, so for the tail section of the curves that we are interested in, uncertainties related to baryonic matter fortunately become subdominant. Therefore, by concentrating on the tail section, it becomes possible to make robust assessments, for which the impact of baryonic matter uncertainty is minimized (if for specific galaxies, high quality data on certain baryonic components are available, it also does not hurt to take them into account explicitly, so as to further reduce the uncertainty, a strategy that we will adopt with our examples below). This lack of contamination by baryonic matter uncertainties is fortunate, since our aim in this paper is precisely to explain, with the EWM, this rising tail end, which is the feature that is in strong conflict with CDM and MOND.

Specifically, to gauge quantitatively how well the EWM profile performs, we note that Equation (5) predicts a linearly-rising EWM contribution to the rotation curve that, in addition, must pass through the origin (i.e., $v=0$ when $\rho=0$ ). In other words, while even the simplest linear fit to the tail will produce two parameters, the slope and the intercept, the EWM profile is so rigid that it only has one parameter $\kappa$ that can be adjusted. This "over-determinacy" translates into a rather stringent test for the EWM profile; if not correct, there is no way it will match both the slope and the intercept of the linear fit with a single parameter. We can also produce a quantitative measurement of this statement, namely by how much the slope of the EWM fit (varying only $\kappa$ ) differs from the slope of the best linear fit (varying both slope and intercept).

Although not the main focus of this paper, definitive assessments can also be made for the inner regions, even without prescribing specific models (thereby bringing in unwarranted assumptions) for the uncertain baryonic matter. After already fixing the definitive baryonic matter and EWM 
contributions, we place the remaining baryonic contribution that would produce a perfect fit (so the blue curve threading the observational points is the overall fitting curve if one prefers) into the "remaining baryonic" component in Figure 1. Definitive physical constrains exist for this left-over (it is not the fitting error and is supposed to be large since all the disc stars, etc., are in it), namely that it should have a shape resembling that arising from a disk occupying the small galactocentric radii regions. More stringently, this contribution has to be a real, as opposed to imaginary, number. In other words, the EWM plus definitive baryonic contribution should not have already overshot the observed curve, leaving negative headroom for the uncertain baryonic matter to contribute.

We begin with the nearby (thus good data quality) M31 (Andromeda). Our detailed fitting procedure is as follows

1. Because M31 exhibits a strong bulge influence, it becomes possible to infer a bulge mass by fitting to the very inner part $(\rho<5 \mathrm{kpc})$ of the rotation curve. This is done because as discussed in the last paragraph, any reduction in the uncertainty about baryonic contributions will further reduce the errors when we fit to the tail section. The bulge contribution is modeled as a mass monopole $\Phi_{\mathrm{Nb}}=-M / \rho$, and by inspection, we obtain $M \approx 4 \times 10^{10} \mathrm{M}_{\odot}$. See Figure 1 a for the fitting results.

2. We then turn to the tail section (beyond the dotted vertical line in Figure 1a at around $33 \mathrm{kpc}$ ). We begin by taking off the monopolar bulge contribution, so that the remainder contains the contribution from the EWM (dominate) and the other non-bulge baryonic components (subdominant).

3. A fit for $\kappa$ is then carried out. Equation (5) predicts an EWM contribution to the tail of the rotation curve at $v=c \sqrt{\kappa} \rho$ (where the speed of light $c$ is in units of $\mathrm{km} / \mathrm{s}$ ), and we fit this functional form to the monopole-removed tail section of the rotation curve. The fitting is done using the standard computation package Mathematica, yielding the best fit $\kappa=5.035 \times 10^{-10} \mathrm{kpc}^{-2}$. For comparison with a full linear fit, it is more convenient to present the result in terms of the overall slope $c \sqrt{\kappa}$, whose best fit value is 6.732, with standard error 0.0498 .

4. The highly rigid nature of the EWM profile (containing only one free parameter) as compared to CDM profiles (containing many tunable parameters) allows us to carry out a test on whether its rigid functional form matches data. Namely, as discussed previously, we can carry out a full linear fit to the tail with $v=a \rho+b$ and see whether $a$ is close to the fit for $c \sqrt{\kappa}$ we obtained during the last step, as well as whether $b$ is close to zero. Recall though that the tail still contains non-bulge baryonic contributions, and they would contribute to $b$; nevertheless, since they are subdominant in the tail section, $b$ should not be large. Our fitting yields the best fit slope at $a=5.96$, with standard error 1.29 , which matches rather well with the $c \sqrt{\kappa}$ obtained earlier (well within one standard error). The parameter $b$ has a best fit value of 27.65 and a standard error of 46.36, which is also consistent with a nearly-vanishing intercept.

5. Finally, a few words regarding the mid-section of the rotation curve are in order. The remaining miscellaneous contributions, from the stars, dust, and gas residing outside of the central core, are collected into the "remaining baryonic" in Figure 1a. We caution that the study by Bournaud et al. [49] on recycled dwarf galaxies suggests the presence of large amounts of difficult-to-see cold molecular gas in the discs of their parent spiral galaxies (see also, e.g., Li et al. [50] for more direct observational evidences); thus, there is likely still an invisible matter component in the aDM (but of a mundane variety; being ordinary matter, their amounts and distributions could also differ substantially between galaxies of different types and ages, just as stellar matter would) that contributes to the inner to intermediate regions of the rotation curves.

Note further that all these aforementioned components are spread out on a disc, and not distributed in a spherically-symmetric manner, so the "remaining baryonic" curve is not bounded from below by a Keplerian profile. Instead, a rapid $\sim 1 / \Delta \rho$ decline near a high matter density strip, $\Delta \rho$ being the distance to the sharp edge of the strip, provides a better approximation 
(and a slower decline in the orbiting speed is to be expected if the drop-off in density is more gradual), and is consistent with Figure 1a.

Beyond bulgy disc galaxies like M31, it is also interesting to examine dwarf galaxies. Because they are aDM-dominated, their entire curves should behave much like the tail, continuously rising throughout the available data range, a trend that is indeed seen in, e.g., Figure 57 of de Blok et al. [46]. Here, we examine in more detail two galaxies investigated by Oh et al. [48], which had been careful to scrub the contaminations due to non-circular motion (a significant problem with dwarfs) from their rotation curves. Beginning with NGC 2366 (Figure 1b), we note that it exhibits a pronounced tail section, which is once again well accounted for by the EWM profile. In contrast, even empirical CDM profiles proposed to resolve the cuspy halo problem cannot fit to it (see Figure 22 in Oh et al. [48]). Our detailed fitting procedure follows closely that of M31 and is as follows:

1. The rotation curve does not contain a clear bulge-dominated inner segment, so we cannot infer a bulge monopole size, but this also means the bulge should not be significant (recall that we are dealing with a dwarf galaxy). Instead, the carefully-analyzed gas contribution derived from the integrated HI map is available for this galaxy, so we explicitly account for this contribution.

2. Turning to the tail section, we once again remove the contribution from the known baryonic component, gas this time, so as to scrub down the tail maximally to a cleaner EWM domination. We then fit for $\kappa$ with the same procedure as was done with M31, yielding best fit $\kappa$ at $6.215 \times 10^{-10} \mathrm{kpc}^{-2}$. Note that despite the drastically different nature of the galaxies (massive spiral versus dwarf) and different rotation speeds (hundreds of $\mathrm{km} / \mathrm{s}$ versus dozens), the $\kappa$ values are remarkably similar between NGC 2366 and M31, differing by only $25 \%$ rather than orders of magnitude.

3. The $\kappa$ value translates into a slope $c \sqrt{\kappa}$ at 7.47916, with standard error 0.0518 . The full linear fit, on the other hand, yields a slope of $a=6.907$ with standard error 0.600 , as well as an intercept $b=4.169$ with standard error 4.356. Once again, the EWM's rigid form is consistent with the morphology of the tail section of the rotation curve ( $a$ and $c \sqrt{\kappa}$ agree within one standard error, and $b$ is consistent with being nearly zero).

4. The uncertain stellar contributions for this faint dwarf galaxy are collected into the "remaining baryonic" contribution.

For the fainter IC 2574 (Figure 1c), we need a somewhat different fitting procedure:

1. Once again, the gas contribution from the integrated HI map is available. However, this time, the gas contribution rises very rapidly on the outer regions, so we end up with a large and varying gas contribution in the tail. This unfortunately obscures the linear EWM tail there (the curve bends downwards following the trend in the gas contribution). Nevertheless, the gas contribution declines at the extreme large $\rho$ end, while EWM keeps rising to greater dominance there, so we can adopt a simpler procedure by letting the EWM match the last point in the rotation curve. This yields $\kappa=4.5 \times 10^{-10} \mathrm{kpc}^{-2}$, differing from the value for M31 by only around $10 \%$, despite the very different galaxy types and rotation curve morphology.

2. The very different (as compared to Figure 1a for massive spiral galaxies), continuously-rising rotation curve morphology (also more or less shared by NGC 2366) seen for the dwarfs is because for these aDM-dominated dwarf galaxies, the EWM contribution is significant throughout the entire curve, even on the inside. The almost linearly-rising curves thus provide a rather direct support for the rigid linear EWM profile. In contrast, they pose a serious challenge to common theoretical CDM profiles that flatten off at large $\rho$, as they cannot provide sufficient speed on the outside without overshooting the inside. Since it is difficult to produce outward acceleration, overshooting is a more troublesome problem. In Figure 1c, we provide a demonstration of this difficulty by making a Navarro-Frenk-White [41] profile fit as a green curve. The fitting is done by 
inspection of the tail of the curve beyond $\rho \sim 7 \mathrm{kpc}$, yielding parameter values $v_{200}=160 \mathrm{~km} / \mathrm{s}$, $\mathcal{C}=1$, and $R_{200}=100 \mathrm{kpc}$, feeding into:

$$
v_{\mathrm{NFW}}(\rho)=v_{200} \sqrt{\frac{\ln (1+\mathcal{C} x)-\mathcal{C} x /(1+\mathcal{C} x)}{x(\ln (1+\mathcal{C})-\mathcal{C} /(1+\mathcal{C}))}}
$$

where $x \equiv \rho / R_{200}$. Note that even though we have tried to suppress the overshooting by reducing $\mathcal{C}$ to a perhaps unrealistically small value (it is around 10-15 for the Milky Way) and by making $R_{200}$ very large (beyond this value, the CDM profile turns downwards), the inner regions of the $\mathrm{CDM}$ predicted curve still rest significantly above the observed rotation curve.

As already alluded to, the $\kappa$ values for all three galaxies are quite similar, which could be understood in the EWM context as due to $\kappa$ being determined by the fairly common general conditions in a local neighborhood of the cluster that set the boundary conditions for the individual galactic halos (both dwarf galaxies reside in the nearby M81 group, and M31 is in our local group; furthermore, the supra-galactic structures within the local group-e.g., planes of dwarf galaxies-have been shown to possess curious alignments [51], so the individual galaxies must all subsume into a common larger cluster-scale structure). We obviously cannot claim a quasi-universality of $\kappa$ based on data from only three galaxies (the scrubbing technique of Oh et al. [48] should be applied to more galaxies to boost the statistics), but nevertheless note that it would be quite a coincidence if the commonality shared by these three rather arbitrarily-chosen cases is purely accidental, given that the galaxies belong to different classes, as well as exhibit different rotation curve morphologies and average speeds. Even more fortunately, establishing the universality of $\kappa$ does not require us to obtain the full rotation curves. At very large galactocentric radii, EWM would dominate, leading to a linear $v=\sqrt{\kappa} \rho$ and thus a constant angular velocity $\sqrt{\kappa}$ (using our average $\kappa \approx 5.3 \times 10^{-10} \mathrm{kpc}^{-2}$ from the three galaxies examined above, this translates into a rotation period for the outer rims of galaxies at about $0.89 \mathrm{Gyr}$ ). Therefore, with HI and other measurements of the outer regions of the galaxies, one should record a universal rotation period, shared between all galaxies regardless of their masses and the radii at which we happen to take readings observationally (such universality would not be present with truly asymptotically flat rotation curves), which matches and provides a robust (without needing additional assumptions such as sharp truncations of the discs) explanation for the observational results reported in Meurer et al. [52] for large sample sizes. Alternatively, recall that the EWM profile resembles that of a spherical CDM halo with a constant density proportional to $\sqrt{\kappa}$ (i.e., an extended core); the universality of $\kappa$ across different galaxies then implies that the characteristic volume density (the overall scaling factor) in best CDM fits should be roughly constant, which is indeed observed to be the case for samples spanning over five decades of galaxy luminosity [53].

Furthermore, this universality in $\kappa$ also provides a simple explanation for the well-known relationship between specific angular momentum $j=J / M$ of a galaxy and its total stellar mass $M$, namely that $j \propto M^{0.6}$ [54]. In the present consideration, we have that, for measurements where baryonic influence does not overwhelm the EWM contribution to the rotation curve (failure of this simplifying condition feeds into the rather significant scattering in the observed $j$ vs. $M$ relation), we can approximate the galaxy as a rigidly-rotating disk with angular velocity $\sqrt{\kappa}$ and some distribution of stellar mass density, say exponential $\rho_{*}=\rho_{\text {core }} \exp (\rho / H)$, where $H$ is some scale distance. Then, simple integration gives:

$$
j=\frac{6}{6.28} \frac{M \sqrt{\kappa}}{\rho_{\text {core }}},
$$

where the central stellar mass density $\rho_{\text {core }}$ is unsurprisingly dependent on $M$, as well, with $\rho_{\text {core }} \propto$ $M^{0.42-0.74}$ observed for early-type galaxies [55]. Therefore, despite extreme crudeness, our estimate can already produce a $M^{0.6}$ power law, while it should be clear as well that, if the angular velocities of galaxies are completely arbitrary, we would not be able to obtain a clean dependence on $M$ alone. 
Note also that the functional form of Equation (7) is not sensitive to the detailed morphology of the galaxy due to dimensionality, but the precise numerical coefficient is, which implies that disc and bulge-dominated galaxies should naturally lie on two different parallel lines in a $\log j$ vs. $\log M$ plot, as is indeed observed.

Another simple estimate shows that the universality of $\kappa$ also leads to the baryonic Tully-Fisher relation [56]. Note first that the flat part of the rotation curve corresponds to where the decline and increase in $v$ arising from the baryonic and EWM contributions balance out, leading to (at sufficiently large $\rho$, the bulge and disc contribution to $v$ can be approximated by a $1 / \sqrt{\rho}$ profile):

$$
\frac{d v}{d \rho}=\left.\frac{d}{d \rho} \sqrt{\frac{G M_{\mathrm{bar}}}{\rho}+c^{2} \kappa \rho^{2}}\right|_{\rho=\rho_{\mathrm{f}}}=0,
$$

where $\rho_{\mathrm{f}}$ signifies the flat part of the rotation curve. Solving for $\rho_{\mathrm{f}}$ and substituting into the rotation speed for that flat region, we have then:

$$
\begin{aligned}
v_{\mathrm{f}} & \left.\equiv \sqrt{\frac{G M_{\mathrm{bar}}}{\rho}+c^{2} \kappa \rho^{2}}\right|_{\rho=\rho_{\mathrm{f}}} \\
& =\frac{\sqrt{3} c^{1 / 3}}{2^{1 / 3}} \kappa^{1 / 6}\left(G M_{\mathrm{bar}}\right)^{1 / 3},
\end{aligned}
$$

or in other words, $M_{\mathrm{bar}} \propto v_{\mathrm{f}}^{3}$ when $\kappa$ is common across galaxies. The observational value for the power index in the relationship between $M_{\mathrm{bar}}$ and $v_{\mathrm{f}}$ is currently under active debate, with the claimed results landing between three (e.g., Gurovich et al. [57] gives a range of $3.0 \pm 0.2$, and the recent study by Ponomareva et al. [58] yields 2.99 \pm 0.2 ) and four (see, e.g., McGaugh [59]). The difference is largely attributable to how the mass-to-light ratio effects are handled, and the ongoing effort to extract a more accurate value is driven by the fact that these two values correspond to those anticipated under CDM and MOND, respectively. Our prediction is more in line with CDM, but if a definitive consensus value of four is eventually reached, it would serve to falsify our proposed model.

\subsection{Off the Galactic Plane}

A very distinguishing feature of the EWM as described (to lowest order approximation) by Equation (5) is the fact that the EWM is "repulsive" off the galactic plane. More specifically, $g_{z}$ is pointed away from the galactic plane, with an increasing strength at larger $|z|$, so it tends to (very slowly; the typical value for EWM acceleration is $\kappa \times 1 \mathrm{kpc} \sim 10^{-12} \mathrm{~ms}^{-2}$ in SI units) remove matter that is not close to this plane or become dislocated from it (e.g., during galactic mergers); thus, it may possibly help to chisel out and maintain super thin pure-disc galaxies through the many mergers that they likely would have experienced (in order to grow to their present sizes), which the CDM framework struggles to account for $[9,60]$.

Note though that the gravitational pull from regular disc matter points in the opposite direction to $g_{z}$, so the stable disc plane is thickened. As some of that disc matter is dim and possibly lumped into aDM contribution, one may even on occasions infer that there is an overall dark attractive force towards the disc. The relative weakness of the "repulsive" $g_{z}$ is even more pronounced in the core region, where the high concentration of ordinary matter dominates (see Figure 2 below). EWM thus contributes little to bulge dynamics and certainly would not send the bulge matter flying away (large elliptical galaxies share similar dynamical characteristics to the bulges of disc galaxies, and their older age likely implies greater proportions of dim ordinary matter-as molecular gases, as well as compact objects-whose distribution and density are unfortunately uncertain). In short, absent major events such as mergers knocking (mostly gaseous) matter into high $|z|$ regions, one should not expect to see significant outflows away from the galaxy when it is resting in a quiescent state.

Even during extreme events such as mergers, the dislocated material does not simply launch vertically into deep space. The combined acceleration directions from the EWM and the massive core of 
ordinary matter is plotted in Figure 2. We see that there are two regimes": (1) matter starting off closer to the disc gets pushed towards the galactic center, but does so by first being carried to higher latitudes and then compressed nearly radially towards the galactic core; (2) matter starting off/knocked out sufficiently far from the disc would eventually get launched into deep space (which possibly explains how metals arrive at large distances of over $150 \mathrm{kpc}$ from the galactic centers [61]), but in a direction more closely hugging the rotation axis of the galaxy.

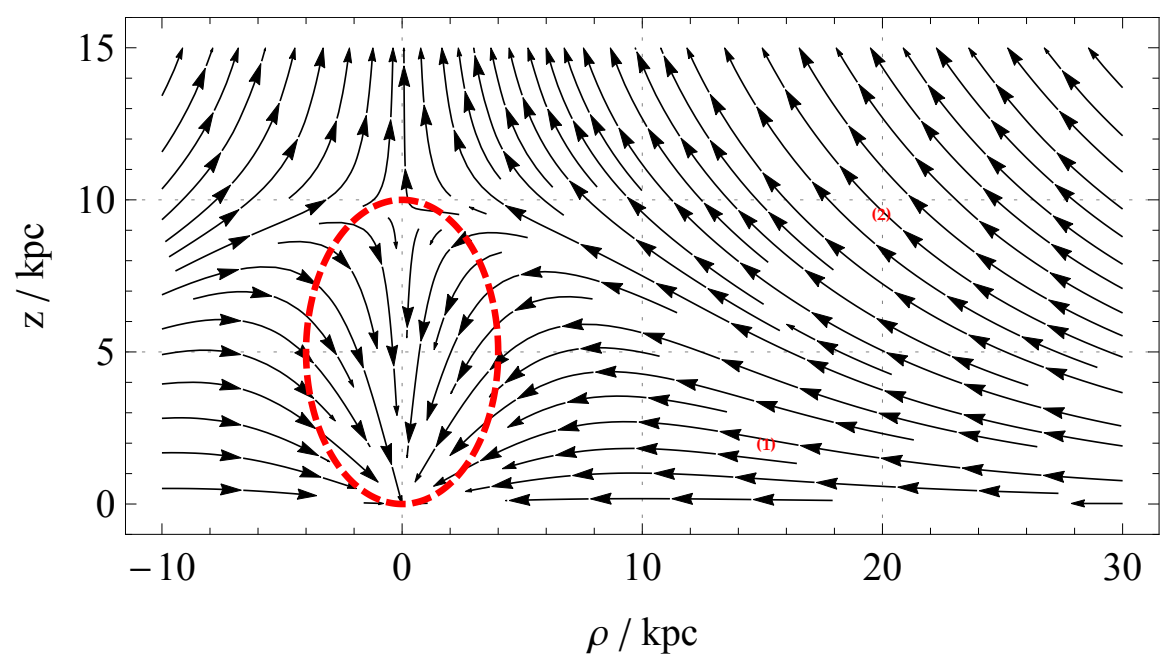

Figure 2. The streamlines of the combined acceleration acting on off-galactic-plane particles, due to the galactic core mass $M$ and the EWM parametrized by $\kappa$. The values of these parameters are taken to approximate the Milky Way, with $\kappa=5.3 \times 10^{-10} \mathrm{kpc}^{-2}$ being the average of the three galaxies from the last section (in the same cluster as the Milky Way) and $M=2 \times 10^{10} \mathrm{M}_{\odot}$, since the overall mass of the Milky Way is about half of that of M31. The red dashed circle signifies the boundary of the Fermi bubble. The coincidence between the bubble and separatrix heights may be accidental, or alternatively, the hardness of the X-ray emissions (Loop I, residing further out, has a softer spectrum) may depend on whether the EWM-driven ambient and active galactic nucleus (AGN)/starburst-driven burst flows are counter-streaming (relevant for shock wave properties).

Spiral galaxies such as the Milky Way would frequently experience minor mergers with dwarf galaxies, during which stars (and gas) from the spiral galaxy would inevitably become dislocated off the disc and into region (1), and subsequently become lifted to moderately high galactic latitudes by the EWM field. The region (1) halo stars thus produced should, and have in fact been observed to, retain some features indicative of their disc ancestry, e.g., in their chemical composition [62], and prograde rotation [63]. Much of the stellar debris originating in the tidally-disrupted dwarf galaxy will however fall into region (2) and form a distinct outer population of halo stars with lower metallicity. The existence of this dichotomy of halo star populations is supported by observations (see e.g., Carollo et al. [63], Hartwick [64] and references therein), and the boundary between their spatial distributions is indeed located at around 10-15 kpc from the galactic center, matching that dividing EWM regions (1) and (2) shown in Figure 2 (i.e., the two EWM regions correspond to the observed inner and outer halos, respectively).

In the radial direction, the stars and gas knocked into Region (1) are guided towards the galactic center, helping to feed the growth of the supermassive black hole, leading to the consequence of an under-abundance of luminous matter in the disc as compared to the sizes of the supermassive black holes, which would then appear more massive than expected from a traditional CDM-based

4 Note that for simplicity, we had not included the matter contribution from the extended disc. Including it will not change the basic picture apart from pushing the separatrix streamline further up, enhancing the size of Regime (1). The plot also does not include the centrifugal forces arising from any circular motion, which would be particle specific. 
co-evolution scenario (see, e.g., Wu et al. [65]). Because merger events are episodic, the galactic core is fed material in discrete bursts. This could provide clues to the missing pieces in the formation mechanism of the mysterious Fermi bubbles [66,67]. These are giant structures seen above and below the galactic center (schematically marked out by the red dashed line in Figure 2) in $\gamma$-rays, microwave, and polarized radio signals [68-70], as well as in X-rays [71]. Digging through the archaeological evidence found within the bubbles, it has been suggested that they are outflows driven by a single past episode of active galactic nucleus (AGN) outburst and/or starburst activity occurring in the galactic core a few million years ago $[66,72,73]$. However, there is currently no contemplation as to where the extra material falling into Sgr $A^{*}$ and/or driving the star formation activities came from in the first place and why it is supplied in a short discrete burst. We note though that there is evidence for several recent minor mergers where the Milky Way had absorbed smaller galaxies, and when driven by the EWM, dislocated gases will take at least (centrifugal forces will likely prolong the journey) a quarter of a billion years to arrive at the galactic core, largely regardless of where they started off (solution to $d^{2} \rho / d t^{2}=-\kappa \rho$ is $\rho=\rho_{0} \cos (\sqrt{\kappa} t)$, so $\rho=0$ when $t=\pi /(2 \sqrt{\kappa})$, independent of initial $\rho_{0}$ ). Therefore, an initially-diffuse plume will automatically compactify into a small extent in $\rho$ and feed either or both of the aforementioned AGN and starburst activities in a bursty fashion. Furthermore, this timetable is broadly in line with, e.g., the previous crossing of the Milky Way's galactic disc by the Sagittarius dwarf galaxy (estimated to have happened around 0.85 Gyr ago; see Purcell et al. [74]).

In addition, gravitational lensing may also provide clues to the structure of the aDM off of the galactic plane, if the lens galaxy is viewed edge-on. Figure 2 shows that the EWM should produce a quadrupolar contribution to the overall lensing potential (the acceleration field is the gradient to the potential; also recall that Figure 2 includes a monopolar contribution from the galactic core, which should be removed for the present consideration), essentially acting as an enhancement to the disc contribution (a monopole plus a quadrupole at the lowest orders), which may be powerful enough to resolve the radio flux-ratio anomalies. Specifically, the brightnesses of the multiple images of a lensed object often do not match traditional ellipsoidal CDM predictions based on the locations of the images (see, e.g., Goobar et al. [75] for an arbitrary example). This discrepancy is difficult to explain even when additional dark substructures are introduced into the modeling of the lens galaxies [76], as the CDM predicted substructure abundance is insufficient to account for the high incidence rate of such anomalies. It has been noted though by Hsueh et al. [77-79] that an edge-on disc can possibly help make up for the residual, provided the discs are massive enough (such masses are currently observationally unavailable). The present consideration offers another alternative, namely that the smooth galactic EWM, behaving much like a very massive disc in terms of producing higher multipoles in the lens potential, accounts for most of the anomalies (other factors such as free-free absorption make up the remainder), leaving little need for injecting additional low mass dark substructures.

\section{Conclusions}

Galaxy rotation curves exhibit features that require gravitational contributions beyond those arising from Newton's law of universal gravitation. Through concentrating on places most directly reflective of said new ingredients, we demonstrate in this paper that they can possibly be introduced within our verified understanding of gravity and particle physics, by noting that the gravitational field, like in any other field theories, does not always require source terms to exist; it can also be sustained by non-trivial boundary and/or initial conditions. On even larger length scales, the loss of symmetries poses a technical challenge to the analytical analysis of the EWM, but constructs such as the integral curves of the Weyl tensor's characteristic directions [80] may provide useful tools for extracting general attributes, e.g., along the lines of [81]. On a broader note, as any numerical relativist working on binary black hole mergers would attest, gravity is not always a surrendering slave to matter (black holes can be excised from the computational domain since no information comes out, and the violently twitching exterior vacuum is what is being simulated), and if some of the aDM turns out to indeed be the EWM, the reverse may in fact be more true of our universe. Substantial efforts likely need to be invested 
to ascertain whether this is indeed the case, with the first steps perhaps being the establishment of whether a rising tail is indeed a universal feature among all galaxies, by carefully examining their large galactocentric radius regions with the next generation of more powerful radio telescopes. The present paper aims to solicit interest from experts of diverse backgrounds to investigate the crevices where GR may be hiding further surprises for us, resulting in the more nuanced features of this more mundane gravity theory having to be considered in place or in addition to other DM considerations.

We must however avoid being overly optimistic and offer up a large dose of caution. The alternative, more established, CDM and MOND proposals have each gone through substantial amounts of scrutiny, and tremendous amounts of efforts have gone into resolving any difficulties they may face. In contrast, the present paper only handles a very specific phenomenology, with no attempt to explain things such as structure formation and other cosmological observations. These questions will have to be answered with extensive studies in the future, by experts in various fields who are more capable than the author, with results that may instantly disprove the proposed model. The present paper can only hope to generate some interest in carrying out those studies, and many more studies are required for the proposed model to be deemed viable at all.

Particularly challenging for the EWM model would be structure formation. This is because the autonomous gravity would behave rather differently from CDM (if one adopts a particle view of gravity, gravitons would be massless, but these massless particles are not like neutrinos or other hot dark matter, since they directly impress long-range forces-gravity-on ordinary matter). It is vitally important that the aDM and ordinary matter couple closely, so that the aDM halo and galaxies are at least spatially colocated. We believe that the autonomous gravitational field would still couple quite directly and closely with ordinary matter, because even though the autonomous gravitational field is not sourced by any matter, it would exert force and accelerate matter just as any gravitational field would. Therefore, just as CDM would attract ordinary matter into the potential well it creates, matter would also sink into any potential well in the autonomous gravitational field and arrange itself to align with the structures of the latter (so the EWM fields would still be centered on galaxies).

On the other hand, the feedback of ordinary matter to the autonomous gravitational field would be much weaker than for CDM and certainly MOND. In principal, GR is nonlinear, so ordinary matter can influence the EWM via the nonlinear coupling between the autonomous field and the gravitational field that the matter sources. However, at the galactic scales, both fields are weak in strength, and the coupling may need a very long accumulation timescale to produce anything significant at all. In contrast, the matter-sourced gravitational field would directly accelerate CDM particles (and pick up MOND corrections even more directly) and thus produce a much stronger feedback. We do not know if strong feedback is still required in the EWM context, since, e.g., we do not have the cuspy halo problem (the EWM profile is like a very extended core; there is not the large amounts of pressure-less CDM-like particles sinking into the center), so the viability of the autonomous gravity proposal in the structure formation context is essentially a very open question.

Funding: The author is supported by the National Natural Science Foundation of China Grants 11503003 and 11633001, the Strategic Priority Research Program of the Chinese Academy of Sciences Grant No. XDB23000000, the Fundamental Research Funds for the Central Universities Grant 2015KJJCB06, and a Returned Overseas Chinese Scholars Foundation grant.

Acknowledgments: We are grateful to several anonymous referees for their insightful comments, which led to many additional discussions, clarifications, and cautions in the paper, which substantially improved the quality and readability of the paper.

Conflicts of Interest: The author declares no conflict of interest.

\section{References}

1. Akerib, D.S.; Alsum, S.; Araújo, H.M.; Bai, X.; Bailey, A.J.; Balajthy, J.; Beltrame, P.; Bernard, E.P.; Bernstein, A.; Biesiadzinski, T.P.; et al. Results from a Search for Dark Matter in the Complete LUX Exposure. Phys. Rev. Lett. 2017, 118, 021303. [CrossRef] [PubMed] 
2. Aprile, E.; Aalbers, J.; Agostini, F.; Alfonsi, M.; Amaro, F.D.; Anthony, M.; Arneodo, F.; Barrow, P.; Baudis, L.; Bauermeister, B.; et al. First Dark Matter Search Results from the XENON1T Experiment. Phys. Rev. Lett. 2017, 119, 181301. [CrossRef] [PubMed]

3. Cui, X.; Abdukerim, A.; Chen, W.; Chen, X.; Chen, Y.; Dong, B.; Fang, D.; Fu, C.; Giboni, K.; Giuliani, F.; et al. Dark Matter Results From 54-Ton-Day Exposure of PandaX-II Experiment. arXiv 2017, arXiv:1708.06917.

4. Ackermann, M.; Ajello, M.; Albert, A.; Atwood, W.B.; Baldini, L.; Ballet, J.; Barbiellini, G.; Bastieri, D.; Bellazzini, R.; Bissaldi, E.; et al. The Fermi Galactic Center GeV Excess and Implications for Dark Matter. Astrophys. J. 2017, 840, 43. [CrossRef]

5. Dicus, D.A.; Kolb, E.W.; Teplitz, V.L.; Wagoner, R.V. Astrophysical bounds on the masses of axions and Higgs particles. Phys. Rev. D 1978, 18, 1829-1834. [CrossRef]

6. Dicus, D.A.; Kolb, E.W.; Teplitz, V.L.; Wagoner, R.V. Astrophysical bounds on very-low-mass axions. Phys. Rev. D 1980, 22, 839-845. [CrossRef]

7. Asztalos, S.J.; Carosi, G.; Hagmann, C.; Kinion, D.; van Bibber, K.; Hotz, M.; Rosenberg, L.J.; Rybka, G.; Hoskins, J.; Hwang, J.; et al. SQUID-Based Microwave Cavity Search for Dark-Matter Axions. Phys. Rev. Lett. 2010, 104, 041301. [CrossRef] [PubMed]

8. Du, N.; Force, N.; Khatiwada, R.; Lentz, E.; Ottens, R.; Rosenberg, L.J.; Rybka, G.; Carosi, G.; Woollett, N.; Bowring, D.; et al. Search for Invisible Axion Dark Matter with the Axion Dark Matter Experiment. Phys. Rev. Lett. 2018, 120, 151301. [CrossRef]

9. Navarro, J.F.; Benz, W. Dynamics of cooling gas in galactic dark halos. Astrophys. J. 1991, 380, $320-329$. [CrossRef]

10. MacCrann, N.; Zuntz, J.; Bridle, S.; Jain, B.; Becker, M.R. Cosmic discordance: Are Planck CMB and CFHTLenS weak lensing measurements out of tune? Mon. Not. R. Astron. Soc. 2015, 451, 2877-2888. [CrossRef]

11. Klypin, A.; Kravtsov, A.V.; Valenzuela, O.; Prada, F. Where Are the Missing Galactic Satellites? Astrophys. J. 1999, 522, 82-92. [CrossRef]

12. Shannon, R.M.; Ravi, V.; Coles, W.A.; Hobbs, G.; Keith, M.J.; Manchester, R.N.; Wyithe, J.S.B.; Bailes, M.; Bhat, N.D.R.; Burke-Spolaor, S.; et al. Gravitational-wave limits from pulsar timing constrain supermassive black hole evolution. Science 2013, 342, 334-337. [CrossRef] [PubMed]

13. Wald, R. It is Not Easy to Fool Mother Nature With a Modified Theory of Gravity; Workshop on Tests of Gravity and Gravitational Physics: Cleveland, OH, USA, 2009.

14. Yunes, N.; Siemens, X. Gravitational-Wave Tests of General Relativity with Ground-Based Detectors and Pulsar-Timing Arrays. Living Rev. Relativ. 2013, 16, 9. [CrossRef] [PubMed]

15. Seifert, M.D. Stability of spherically symmetric solutions in modified theories of gravity. Phys. Rev. D 2007, 76, 064002. [CrossRef]

16. Famaey, B.; McGaugh, S. Modified Newtonian Dynamics (MOND): Observational Phenomenology and Relativistic Extensions. Living Rev. Relativ. 2012, 15, 10. [CrossRef] [PubMed]

17. de Sitter, W. On Einstein's theory of gravitation and its astronomical consequences. Second paper. Mon. Not. R. Astron. Soc. 1916, 77, 155-184. [CrossRef]

18. Gödel, K. An Example of a New Type of Cosmological Solutions of Einstein's Field Equations of Gravitation. Rev. Mod. Phys. 1949, 21, 447-450. [CrossRef]

19. Brill, D.R.; Hartle, J.B. Method of the Self-Consistent Field in General Relativity and its Application to the Gravitational Geon. Phys. Rev. 1964, 135, 271-278. [CrossRef]

20. Misner, C.W.; Taub, A.H. A Singularity-free Empty Universe. Sov. J. Exp. Theor. Phys. 1969, $28,122$.

21. Calabi, E. The space of Kähler metrics. Proc. Internat. Congr. Math. Amst. 1954, 2, 206-207. [CrossRef]

22. Yau, S.T. On the Ricci curvature of a compact Kähler manifold and the complex Monge-Ampere equation. I. Commun. Pure Appl. Math. 1978, 31, 339-411. [CrossRef]

23. Zhang, F.; Brink, J.; Szilágyi, B.; Lovelace, G. Geometrically motivated coordinate system for exploring spacetime dynamics in numerical-relativity simulations using a quasi-Kinnersley tetrad. Phys. Rev. D 2012, 86, 084020. [CrossRef]

24. Hall, G.S.; MacNay, L. Sectional curvature and the energy momentum tensor. Class. Quantum Gravity 2005, 22, 1493-1502. [CrossRef]

25. Lindblom, L.; Scheel, M.A.; Kidder, L.E.; Pfeiffer, H.P.; Shoemaker, D.; Teukolsky, S.A. Controlling the growth of constraints in hyperbolic evolution systems. Phys. Rev. D 2004, 69, 124025. [CrossRef] 
26. Clowe, D.; Bradač, M.; Gonzalez, A.H.; Markevitch, M.; Randall, S.W.; Jones, C.; Zaritsky, D. A Direct Empirical Proof of the Existence of Dark Matter. Astrophys. J. Lett. 2006, 648, L109-L113. [CrossRef]

27. Jee, M.J.; Hoekstra, H.; Mahdavi, A.; Babul, A. Hubble Space Telescope/Advanced Camera for Surveys Confirmation of the Dark Substructure in A520. Astrophys. J. 2014, 783, 78. [CrossRef]

28. Milosavljević, M.; Koda, J.; Nagai, D.; Nakar, E.; Shapiro, P.R. The Cluster-Merger Shock in 1E 0657-56: Faster than a Speeding Bullet? Astrophys. J. Lett. 2007, 661, L131-L134. [CrossRef]

29. Dawson, W.A.; Wittman, D.; Jee, M.J.; Gee, P.; Hughes, J.P.; Tyson, J.A.; Schmidt, S.; Thorman, P.; Bradač, M.; Miyazaki, S.; et al. Discovery of a Dissociative Galaxy Cluster Merger with Large Physical Separation. Astrophys. J. Lett. 2012, 747, L42. [CrossRef]

30. Alexander, S.; Yunes, N. Chern-Simons Modified General Relativity. Phys. Rept. 2009, 480, 1-55. [CrossRef]

31. Obukhov, Y.N.; Hehl, F.W. On the relation between quadratic and linear curvature Lagrangians in Poincare gauge gravity. Acta Phys. Polon. 1996, B27, 2685-2694.

32. Cherubini, C.; Bini, D.; Capozziello, S.; Ruffini, R. Second order scalar invariants of the Riemann tensor: Applications to black hole space-times. Int. J. Mod. Phys. 2002, D11, 827-841. [CrossRef]

33. Penrose, R.; Rindler, W. Spinors and Space-Time, Volume 2; Cambridge University Press: Cambridge, UK, 1986.

34. van Dokkum, P.; Danieli, S.; Cohen, Y.; Merritt, A.; Romanowsky, A.J.; Abraham, R.; Brodie, J.; Conroy, C.; Lokhorst, D.; Mowla, L.; et al. A galaxy lacking dark matter. Nature 2018, 555, 629-632. [CrossRef]

35. Massey, R.; Kitching, T.; Richard, J. The dark matter of gravitational lensing. Rep. Progr. Phys. 2010, 73, 086901. [CrossRef]

36. Trujillo, I.; Carretero, C.; Patiri, S.G. Detection of the Effect of Cosmological Large-Scale Structure on the Orientation of Galaxies. Astrophys. J. Lett. 2006, 640, L111-L114. [CrossRef]

37. Karachentsev, I.D. Missing dark matter in the local universe. Astrophys. Bull. 2012, 67, 123-134. [CrossRef]

38. de Blok, W.J.G. The Core-Cusp Problem. Adv. Astron. 2010, 2010, 789293. [CrossRef]

39. Kent, S.M. Dark matter in spiral galaxies. II-Galaxies with H I rotation curves. Astron. J. 1987, 93, 816-832. [CrossRef]

40. Corbelli, E.; Lorenzoni, S.; Walterbos, R.; Braun, R.; Thilker, D. A wide-field H I mosaic of Messier 31. II. The disk warp, rotation, and the dark matter halo. Astron. Astrophys. 2010, 511, A89. [CrossRef]

41. Navarro, J.F.; Frenk, C.S.; White, S.D.M. The Structure of Cold Dark Matter Halos. Astrophys. J. 1996, $462,563$. [CrossRef]

42. Navarro, J.F.; Frenk, C.S.; White, S.D.M. A Universal Density Profile from Hierarchical Clustering. Astrophys. J. 1997, 490, 493-508. [CrossRef]

43. Ruiz-Granados, B.; Rubino-Martin, J.A.; Florido, E.; Battaner, E. Magnetic fields and the outer rotation curve of M31. Astrophys. J. 2010, 723, L44. [CrossRef]

44. Milgrom, M. A modification of the Newtonian dynamics as a possible alternative to the hidden mass hypothesis. Astrophys. J. 1983, 270, 365-370. [CrossRef]

45. Chemin, L.; Carignan, C.; Foster, T. H I Kinematics and Dynamics of Messier 31. Astrophys. J. 2009, 705, 1395-1415. [CrossRef]

46. de Blok, W.J.G.; Walter, F.; Brinks, E.; Trachternach, C.; Oh, S.H.; Kennicutt, R.C., Jr. High-Resolution Rotation Curves and Galaxy Mass Models from THINGS. Astron. J. 2008, 136, 2648-2719. [CrossRef]

47. Wojnar, A.; Sporea, C.; Borowiec, A. A Simple Model for Explaining Galaxy Rotation Curves. Galaxies 2018, 6, 70. [CrossRef]

48. Oh, S.H.; de Blok, W.J.G.; Walter, F.; Brinks, E.; Kennicutt, R.C., Jr. High-Resolution Dark Matter Density Profiles of THINGS Dwarf Galaxies: Correcting for Noncircular Motions. Astron. J. 2008, 136, 2761-2781. [CrossRef]

49. Bournaud, F.; Duc, P.A.; Brinks, E.; Boquien, M.; Amram, P.; Lisenfeld, U.; Koribalski, B.S.; Walter, F.; Charmandaris, V. Missing Mass in Collisional Debris from Galaxies. Science 2007, 316, 1166. [CrossRef]

50. Li, D.; Tang, N.; Nguyen, H.; Dawson, J.R.; Heiles, C.; Xu, D.; Pan, Z.; Goldsmith, P.F.; Gibson, S.J.; Murray, C.E.; et al. Where is $\mathrm{OH}$ and Does It Trace the Dark Molecular Gas (DMG)? Astrophys. J. Suppl. 2018, 235, 1. [CrossRef]

51. Pawlowski, M.S.; Kroupa, P.; Jerjen, H. Dwarf galaxy planes: The discovery of symmetric structures in the Local Group. Mon. Not. R. Astron. Soc. 2013, 435, 1928-1957. [CrossRef]

52. Meurer, G.R.; Obreschkow, D.; Wong, O.I.; Zheng, Z.; Audcent-Ross, F.M.; Hanish, D.J. Cosmic clocks: A Tight Radius-Velocity Relationship for HI-Selected Galaxies. arXiv 2018, arXiv:1803.04716. 
53. Li, P.; Lelli, F.; McGaugh, S.S.; Starkman, N.; Schombert, J.M. A constant characteristic volume density of dark matter haloes from SPARC rotation curve fits. arXiv 2018, arXiv:1811.00553.

54. Fall, S.M.; Romanowsky, A.J. Angular Momentum and Galaxy Formation Revisited: Effects of Variable Mass-to-light Ratios. Astrophys. J. Lett. 2013, 769, L26. [CrossRef]

55. Saracco, P.; Gargiulo, A.; Longhetti, M. On the central stellar mass density and the inside-out growth of early-type galaxies. Mon. Not. R. Astron. Soc. 2012, 422, 3107-3117. [CrossRef]

56. McGaugh, S.S.; Schombert, J.M.; Bothun, G.D.; de Blok, W.J.G. The Baryonic Tully-Fisher Relation. Astrophys. J. Lett. 2000, 533, L99-L102. [CrossRef]

57. Gurovich, S.; Freeman, K.; Jerjen, H.; Staveley-Smith, L.; Puerari, I. The Slope of the Baryonic Tully-Fisher Relation. Astron. J. 2010, 140, 663-676. [CrossRef]

58. Ponomareva, A.A.; Verheijen, M.A.W.; Papastergis, E.; Bosma, A.; Peletier, R.F. From light to baryonic mass: the effect of the stellar mass-to-light ratio on the Baryonic Tully-Fisher relation. Mon. Not. R. Astron. Soc. 2018, 474, 4366-4384. [CrossRef]

59. McGaugh, S.S. The Baryonic Tully-Fisher Relation of Gas-rich Galaxies as a Test of $\Lambda$ CDM and MOND. Astron. J. 2012, 143, 40. [CrossRef]

60. Kormendy, J.; Drory, N.; Bender, R.; Cornell, M.E. Bulgeless Giant Galaxies Challenge Our Picture of Galaxy Formation by Hierarchical Clustering. Astrophys. J. 2010, 723, 54-80. [CrossRef]

61. Tumlinson, J.; Thom, C.; Werk, J.K.; Prochaska, J.X.; Tripp, T.M.; Weinberg, D.H.; Peeples, M.S.; O'Meara, J.M.; Oppenheimer, B.D.; Meiring, J.D.; et al. The Large, Oxygen-Rich Halos of Star-Forming Galaxies Are a Major Reservoir of Galactic Metals. Science 2011, 334, 948. [CrossRef] [PubMed]

62. Bergemann, M.; Sesar, B.; Cohen, J.G.; Serenelli, A.M.; Sheffield, A.; Li, T.S.; Casagrande, L.; Johnston, K.V.; Laporte, C.F.P.; Price-Whelan, A.M.; et al. Two chemically similar stellar overdensities on opposite sides of the plane of the Galactic disk. Nature 2018, 555, 334-337. [CrossRef]

63. Carollo, D.; Beers, T.C.; Lee, Y.S.; Chiba, M.; Norris, J.E.; Wilhelm, R.; Sivarani, T.; Marsteller, B.; Munn, J.A.; Bailer-Jones, C.A.L.; et al. Two stellar components in the halo of the Milky Way. Nature 2007, 450, 1020-1025. [CrossRef]

64. Hartwick, F.D.A. The Structure of the Galactic Halo; Gilmore, G., Carswell, B., Eds.; NATO Advanced Science Institutes (ASI) Series C; Springer: Berlin, Germany, 1987; Volume 207, pp. 281-290.

65. Wu, X.B.; Wang, F.; Fan, X.; Yi, W.; Zuo, W.; Bian, F.; Jiang, L.; McGreer, I.D.; Wang, R.; Yang, J.; et al. An ultraluminous quasar with a twelve-billion-solar-mass black hole at redshift 6.30. Nature 2015, 518, 512-515. [CrossRef]

66. Su, M.; Slatyer, T.R.; Finkbeiner, D.P. Giant Gamma-ray Bubbles from Fermi-LAT: Active Galactic Nucleus Activity or Bipolar Galactic Wind? Astrophys. J. 2010, 724, 1044-1082. [CrossRef]

67. Dobler, G.; Finkbeiner, D.P.; Cholis, I.; Slatyer, T.; Weiner, N. The Fermi Haze: A Gamma-ray Counterpart to the Microwave Haze. Astrophys. J. 2010, 717, 825-842. [CrossRef]

68. Finkbeiner, D.P. Microwave Interstellar Medium Emission Observed by the Wilkinson Microwave Anisotropy Probe. Astrophys. J. 2004, 614, 186-193. [CrossRef]

69. Planck Collaboration.; Ade, P.A.R.; Aghanim, N.; Arnaud, M.; Ashdown, M.; Atrio-Barandela, F.; Aumont, J.; Baccigalupi, C.; Balbi, A.; Banday, A.J.; et al. Planck intermediate results. IX. Detection of the Galactic haze with Planck. Astron. Astrophys. 2013, 554, A139. [CrossRef]

70. Carretti, E.; Crocker, R.M.; Staveley-Smith, L.; Haverkorn, M.; Purcell, C.; Gaensler, B.M.; Bernardi, G.; Kesteven, M.J.; Poppi, S. Giant magnetized outflows from the centre of the Milky Way. Nature 2013, 493, 66-69. [CrossRef] [PubMed]

71. Snowden, S.L.; Egger, R.; Freyberg, M.J.; McCammon, D.; Plucinsky, P.P.; Sanders, W.T.; Schmitt, J.H.M.M.; Trümper, J.; Voges, W. ROSAT Survey Diffuse X-Ray Background Maps. II. Astrophys. J. 1997, 485, 125-135. [CrossRef]

72. Ackermann, M.; Albert, A.; Atwood, W.B.; Baldini, L.; Ballet, J.; Barbiellini, G.; Bastieri, D.; Bellazzini, R.; Bissaldi, E.; Blandford, R.D.; et al. The Spectrum and Morphology of the Fermi Bubbles. Astrophys. J. 2014, 793, 64. [CrossRef]

73. Bordoloi, R.; Fox, A.J.; Lockman, F.J.; Wakker, B.P.; Jenkins, E.B.; Savage, B.D.; Hernandez, S.; Tumlinson, J.; Bland-Hawthorn, J.; Kim, T.S. Mapping the Nuclear Outflow of the Milky Way: Studying the Kinematics and Spatial Extent of the Northern Fermi Bubble. Astrophys. J. 2017, 834, 191. [CrossRef] 
74. Purcell, C.W.; Bullock, J.S.; Tollerud, E.J.; Rocha, M.; Chakrabarti, S. The Sagittarius impact as an architect of spirality and outer rings in the Milky Way. Nature 2011, 477, 301-303. [CrossRef]

75. Goobar, A.; Amanullah, R.; Kulkarni, S.R.; Nugent, P.E.; Johansson, J.; Steidel, C.; Law, D.; Mörtsell, E.; Quimby, R.; Blagorodnova, N.; et al. iPTF16geu: A multiply imaged, gravitationally lensed type Ia supernova. Science 2017, 356, 291-295. [CrossRef]

76. Xu, D.; Sluse, D.; Gao, L.; Wang, J.; Frenk, C.; Mao, S.; Schneider, P.; Springel, V. How well can cold dark matter substructures account for the observed radio flux-ratio anomalies. Mon. Not. R. Astron. Soc. 2015, 447, 3189-3206. [CrossRef]

77. Hsueh, J.W.; Fassnacht, C.D.; Vegetti, S.; McKean, J.P.; Spingola, C.; Auger, M.W.; Koopmans, L.V.E.; Lagattuta, D.J. SHARP-II. Mass structure in strong lenses is not necessarily dark matter substructure: A flux ratio anomaly from an edge-on disc in B1555+375. Mon. Not. R. Astron. Soc. 2016, 463, L51-L55. [CrossRef]

78. Hsueh, J.W.; Oldham, L.; Spingola, C.; Vegetti, S.; Fassnacht, C.D.; Auger, M.W.; Koopmans, L.V.E.; McKean, J.P.; Lagattuta, D.J. SHARP-IV. An apparent flux-ratio anomaly resolved by the edge-on disc in B0712+472. Mon. Not. R. Astron. Soc. 2017, 469, 3713-3721. [CrossRef]

79. Hsueh, J.W.; Despali, G.; Vegetti, S.; Xu, D.; Fassnacht, C.D.; Metcalf, R.B. Flux-ratio anomalies from discs and other baryonic structures in the Illustris simulation. Mon. Not. R. Astron. Soc. 2018, 475, $2438-2451$. [CrossRef]

80. Owen, R.; Brink, J.; Chen, Y.; Kaplan, J.D.; Lovelace, G.; Matthews, K.D.; Nichols, D.A.; Scheel, M.A.; Zhang, F.; Zimmerman, A.; et al. Frame-Dragging Vortexes and Tidal Tendexes Attached to Colliding Black Holes: Visualizing the Curvature of Spacetime. Phys. Rev. Lett. 2011, 106, 151101. [CrossRef] [PubMed]

81. Zimmerman, A.; Nichols, D.A.; Zhang, F. Classifying the isolated zeros of asymptotic gravitational radiation by tendex and vortex lines. Phys. Rev. D 2011, 84, 044037. [CrossRef]

(C) 2019 by the authors. Licensee MDPI, Basel, Switzerland. This article is an open access article distributed under the terms and conditions of the Creative Commons Attribution (CC BY) license (http:/ / creativecommons.org/licenses/by/4.0/). 